\title{
Autonomous and context-aware scheduling for public displays using place-based tag clouds
}

\author{
Fernando Reinaldo Ribeiro \\ Informatics Department, Polytechnic Institute of Castelo Branco, Portugal
}

Rui José

Information Systems Department, University of Minho, Portugal

\begin{abstract}
Public displays are an increasingly ubiquitous element of our sociodigital landscape with a strong potential to become key building blocks for AmI. For that purpose, they should have the ability to dynamically and autonomously select content from web sources according to the relevance of their content to the continuous flow of social settings around the display. In this paper, we explore the creation of a public display system that evaluates the relevance of content from web sources and selects the most relevant content according to a dynamic tag cloud that incorporates static place definitions, but is also sensitive to the people around the display. We have developed and evaluate a dynamic content selection system based on those principles. The results show that place visitors recognize the sensitivity of the system to their demands and that a place tag cloud can provide an important element for the interpretation of place and for the combination of the interests expressed by the place owner and the multiple place visitors.
\end{abstract}

\section{Introduction}

In Ambient Intelligence (AmI), smart interfaces can perceive and react to people, sense ongoing human activities and proactively respond to them. Public displays are an increasingly ubiquitous element of our socio-digital landscape with a strong potential to become important building blocks for AmI. However, most public displays today are mere distribution points for pre-defined and centrally created content that is not very valued by their potential users and is often perceived as too institutional or dull [1]. The key reasons for this limited success revolve around the limited content made available or their total unawareness regarding the Human activities taking place at that same location. Likewise, the solution may be found in the ability to dynamically integrate content from the web and select content sources according to their relevance to the social context around the display. 
In this paper, we describe the creation and evaluation of a public display system that autonomously selects from web sources the content deemed more relevant according to a dynamic place model that is sensitive to the people around the display. People can express their content preferences by specifying tags in their Bluetooth device name, as described in [2]. These tags, together with seed tags defined by a place owner constitute a tag cloud that is continuously reflecting the social setting around the display, being sensitive to immediate indications of interest and providing a balanced combination between the content suggestions expressed by multiple place visitors and those expressed by the place owner. This tag cloud is then used as the basis for an adaptive scheduling algorithm that addresses the specific requirements raised by content recommendation in public displays. The results show that place visitors recognize the sensitivity of the system to their demands and that a place tag cloud can provide an important element for the interpretation of place and for combining the dynamic set of interests expressed by multiple people.

\section{Related work}

Determining what to present next is a central feature for any public display system. In commercial systems, this is typically based on a fixed scheduling cycle of pre-defined content. Adaptive scheduling alternatives have been explored that introduce sensibility of the schedule to some type of external variable. Proactive displays [3] recognize specific presences and display information from associated profiles. Context-aware informative display [4] and context sensitive public display for adaptive multi-user information visualization [5] also aim to provide people with relevant content in an opportune and personalized way. Dynamo [6] or BlueBoard [7] are examples of systems that give users direct control of the display and thus content selection is directly handled by users rather than by the system. BlueScreeen [8] bases its scheduling decisions on history observation of exposed Bluetooth devices, which were collected during the advertising cycles. Muller [9] proposes a system that uses a naïve Bayes classifier and information about history, current time, location, advert keywords and feedback to determine the best advert to display. These systems support unassisted and adaptive scheduling, but they employ customized scheduling algorithms based on users' profiles or content selected from a predefined list.

In its essence, the problem addressed in this work shares many objectives with recommendation systems, which are usually classified into three main categories: content-based, collaborative, and hybrid recommendation approaches [10]. A recommendation system is focused on estimating ratings for items that have not been seen by a user. This estimation is usually based on the ratings given by this user to other items or based on ratings given by other users with similar interests. However, the specific scenario of recommending content from web sources on a public 
display raises specific challenges that break some of the assumptions we often find in recommendation algorithms. The first challenge is that in a public display interaction is much more limited. In many cases, the intended use is simply to be seen, which does not generate any information at all about how they are used, much less about the users interests or preferences. Also, because there is normally no intermediate stage between content being suggested and being presented, there are considerably higher risks associated with inappropriate recommendations.

The second challenge is how to recommend content for a place instead of a person. Rather than having a user profile, a public display needs a place profile that combines the preferences of the person managing the display, who we call the place owner, with the preferences of the multiple users that may use the display.

Finally, and because we are considering dynamic content from web sources, we must address the fact that the relevance of the respective information is likely to face considerable oscillations, ranging between rich recently updated information and strongly deprecated or even non existing information. Together, these characteristics raise specific recommendation timings that are not common in most recommendation problems. The notion of most relevant content may change very quickly because of changes in the content itself, changes in competing content or changes on the set of people being sensed. As a consequence, the adaptation processes at the various selection levels must all be very dynamic and frequently reevaluate their selection decisions.

\section{A place-based content scheduler}

A key part of this work was the specification and implementation of a content scheduler, represented in Fig.1, that is able to select the information feeds deemed more relevant for the current social setting.

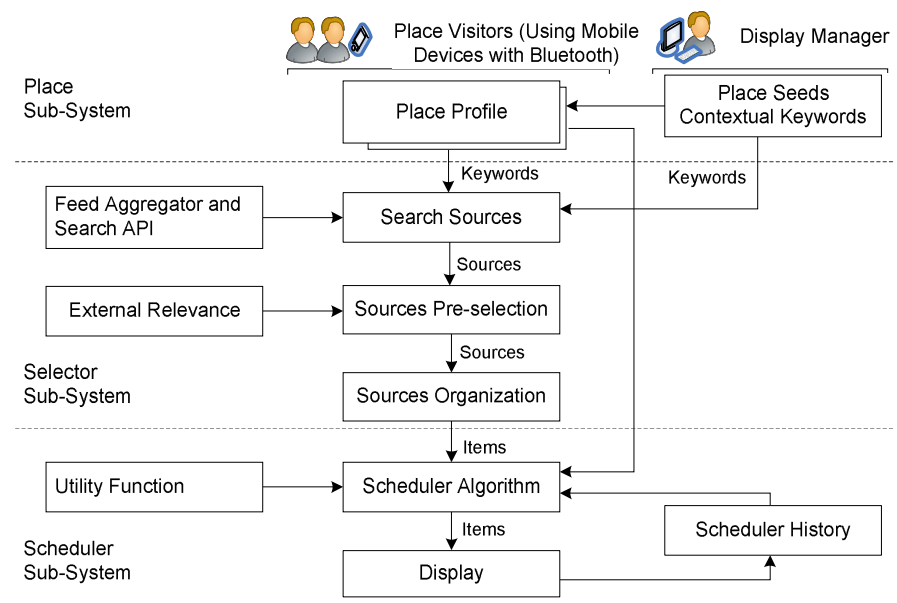

Fig. 1. System Architecture. 
There are three main components in this architecture: A place model that represents a dynamic and evolving view of the place in the form of a tag cloud, a selector that takes the keywords in the tag cloud and retrieves relevant content for each of those tags, and a context-aware scheduler that considers the weight and current presence of the represented tags to select which content is going to be shown next.

Place model. Place is a very broad concept, involving situated practices and a fluid interpretation of the surrounding social context. The model used in this system to represent place is based on a shared and historical view of the expressions of interest made by the people around the display. The model must also combine the expression of interests from a place owner, who is expected to exert stronger influence in the process of place making, and the multiple place visitors. Our approach to these issues is based on a place tag cloud. A tag cloud can combine multiple sources of content preferences, can easily be visualized to support interpretation and facilitates keyword-based content selection. The place tag cloud is first created by the place owner, who may define a set of seed words together with additional parameters that determine if those words always remain in the tag cloud or if they can be replaced by new words as they enter the tag cloud. A list of blocked keywords may also be specified as a simple filter for inappropriate content. Place visitors can also publish their own interests through tag commands in their Bluetooth device name [2]. The combination of multiple contributions from place visitors through time characterizes place as a seamless sequence of many social settings.

Selector. The selector sub-system is responsible for autonomously finding and selecting relevant content sources from the Internet. In this implementation, all the sources are dynamically selected and thus there are no predefined sources. The selection is based on the keywords from the place tag cloud and for each of the keywords the selector, selects the set of content considered more relevant. The selection within each keyword is based on source relevance. This is a non contextual measure and it is calculated as a function of two external indicators: the rank from Alexa Traffic Rank and the number of subscribers of each source. This gives an important marker that can help to improve the system utility, pre-selecting relevant sources and eliminating those sources that no one considers relevant.

Scheduler. The scheduler sub-system selects which of the tags in the place tag cloud will be used next for content presentation. For each tag there is information about the respective popularity and whether or not it is currently present, i.e. someone who is now around the display is announcing that tag. Additionally, there is also a waiting queue with the most recently selected tags. These tags are excluded and they are released again after a few selections. The algorithm selects the most popular tag among those who are present and not in the waiting queue, or if none is present, the most popular tag not in the waiting queue. When a tag is selected, the next step is to select which of the associated content items will be displayed next, using a multi-criteria utility function that considers multiple elements such as the length of the text, the content language, the number of links, and timeliness [11]. 


\section{Evaluation and Discussion}

The evaluation of this system was mostly based on user perception about the system behaviour rather than on its accuracy. It would always be subjective to set any comparison metrics for assessing the appropriateness of content to the social environment around the display. Therefore, we chose to focus on how users perceive the behaviour of the system within their own conceptual frame of the surrounding context. More specifically, our evaluation was focused on the following goals: the appropriateness of the place tag cloud as a shared and public model for the social setting around the display; the viability of tag indications as a simple interaction mechanism; the recognition by place visitors of the sensitivity of the system to their demands; and their overall perception that the content displayed is relevant for the place as expressed by the tag cloud.

\subsection{Experimental work}

To evaluate the recommender system we have conducted a 3 weeks experiment at the main entrance of the Information Systems Department at UMinho. This is mostly a transient place where students, teachers and staff walk by on their way to classes and labs, occasionally forming small groups. The prototype is composed by a large display that includes three panels (see figure 2 ).

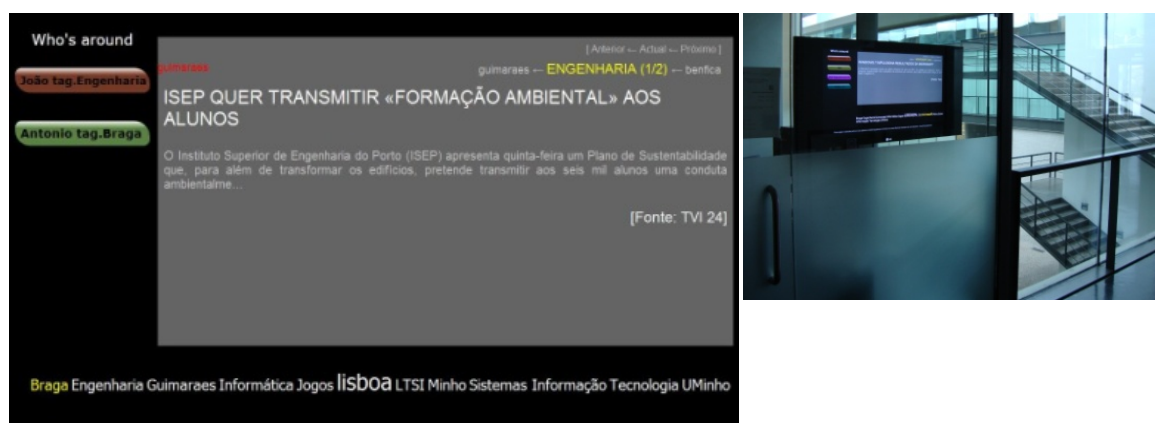

Fig. 2. a) Public display screenshot. b) Prototype image.

The left panel presents Bluetooth device names that are currently detected in the place. This is the "who is around" panel and essentially serves as a first level of presence recognition. People can add tags to their Bluetooth device name. The tag is added to the place tag cloud, if new, and marked as being present. Its popularity will steadily begin to increase while that presence remains. The place cloud is represented in the bottom panel. The size of the tags is proportional to their popularity in the place and keywords that are currently present are shown in yel- 
low as an additional level of presence recognition and interaction feedback. The main panel presents content according to the current place profile. At its top, this panel displays the sequence of keywords being used for content selection, more specifically, the previous, the current and the next tags being used, thus facilitating the interpretation of the system behaviour by users.

For this experiment the display was configured to favour Portuguese content, to avoid presenting the same item every 10 presentations and to avoid present items from the same source every 5 items. The canonical view of the place was set by defining a number of seed tags for the place tag cloud associated with Informatics and Engineering topics as well as location related keywords associated with the town and region. During these 3 weeks we collected usage logs and conducted a total of 15 structured interviews with people at the place who had previously tried to use the system.

\subsection{Results and discussion}

Table 1 summarises the usage statistics observed during the 3 weeks of the experiment between $9 \mathrm{am}$ and $8 \mathrm{pm}$ of the working days.

Table 1. Experiment statistical data.

\begin{tabular}{ll}
\hline Distinct Bluetooth device names detected & 349 \\
Bluetooth devices detected & 308 \\
Total explicit interactions (tag commands across multiple sessions) & 73 \\
Distinct explicit interactions (unique tag commands) & 31 \\
Distinct Bluetooth devices with explicit interactions & 23 \\
Number of scheduled items & 21616 \\
Number of items selected from interactions originated tags & 5567 \\
Number of items selected from tags while those tags were present & 360 \\
Number of selected sources & 128
\end{tabular}

The numbers show that there were 23 devices originating tags to the display. As a result 1,67\% (360) of the scheduled items were presented as a direct reaction to tag publications while the respective user was present. This reflects the relatively limited number of people interacting with the system and above all the transient nature of the place, with most people just stopping for a very short interaction and then moving away. We can also observe that $25,7 \%$ of all schedulings were consequence of users interactions (with the remaining $74,3 \%$ being based on seed tags). This number is particularly interesting because it reflects the influence of the aggregate of interactions in the display behaviour even after the people making the interactions are gone. This number is strongly affected by the interaction patterns, but also by key behaviour parameters such as the decay level in the 
popularity of tags or the minimum popularity defined for seed tags. A quick decay will make the system more responsive to new arriving tags, but it will also fallback more quickly to the seed tags. A slow decay will tend to consolidate more commonly used tags, favouring an emerging view of place, but it will be much more difficult for new tags to reach the new level of popularity as those already in the tag cloud. This is therefore a key parameter in setting the responsiveness of the system and the balance between a pre-defined and a situated view of place.

To evaluate people's perception about the sensitivity of the system to their requests, uncovering opinions about the relevance of the content displayed, its timeliness and responsiveness to indications of interest, we also ask 15 place visitors who had used the systems to answers 6 groups of questions related with their experience. For each group there was also an open question where users are able to make their comments about the topic. Table 2 presents the results organized by groups of questions.

Table 2. Survey summary (group results).

\begin{tabular}{lcccc}
\hline Questions group & Good & Sufficient & Poor & Bad \\
\hline 1) Relevance of the content presented in the display & $48,8 \%$ & $41,9 \%$ & $7,0 \%$ & $2,3 \%$ \\
2) Influence of the interactions in the display behavior & $30,2 \%$ & $58,1 \%$ & $9,3 \%$ & $2,3 \%$ \\
3) Impact and acceptance & $24,1 \%$ & $58,6 \%$ & $17,2 \%$ & $0 \%$ \\
4) Handle with abusive behaviors & $0 \%$ & $83,3 \%$ & $0 \%$ & $17,2 \%$ \\
5) Interactions & $32,5 \%$ & $45,5 \%$ & $15,6 \%$ & $6,5 \%$ \\
6) Privacy & $76,9 \%$ & $0 \%$ & $23,1 \%$ & $0 \%$ \\
\hline
\end{tabular}

Overall, these results indicate that the perception of users in regard to the behaviour of the system was mainly positive. Some users, however, considered the system reaction to be slow as they seemed to expect an almost immediate change in content when defining new tags. While an immediate response was never our goal and even considering that setting the right expectations may be enough to change user perception regarding this issue, the relatively slow responsiveness may still represent a real limitation to the use of this approach in transient places.

\section{Conclusions}

In this work, we explored the usage of a dynamic scheduling model for content selection for situated public displays. This model is supported by a shared and public place profile in the form of a place tag cloud that combines the multiple interests of the place owner and place visitors. The positive results obtained during the evaluation suggest that this is a viable approach to the problem of selecting relevant content for a dynamic view of place. In particular, the visual nature of the tag cloud seems to facilitate the interpretation of the system behaviour in a way that influences positively the user perception, even when the selections are not perfect. 
Further research is needed to evaluate across multiple settings the ideal values for some of the system parameters. For example the decay of user-suggested tags affects responsiveness and also the balance between pre-defined and emerging notions of place, while the size of non-repetition queues affects the balance between content quality and diversity. Overall, these results suggest that this may be a valuable step towards the emergence of dynamic place profiles that match the social expectations and practices of their evolving social setting.

Acknowledgments The first author was supported by a Portuguese Foundation for Science and Technology scholarship (SFRH/BD/31292/2006).

\section{References}

[1] E. M. Huang, A. Koster, and J. Borchers. (2009). Overcoming Assumptions and Uncovering Practices: When Does the Public Really Look at Public Displays?. 6th International Conference on Pervasive Computing. Sydney, Australia.

[2] R. José, N. Otero, S. Izadi, and R. Harper. (2008). Instant Places: Using Bluetooth for Situated Interaction in Public Displays. IEEE Pervasive Computing, vol. 7. pp. 52-57.

[3] D. W. McDonald, J. F. McCarthy, S. Soroczak, D. H. Nguyen, and A. M. Rashid. (2008). Proactive Displays: Supporting Awareness in Fluid Social Environments. ACM Transactions on Computer-Human Interaction. vol. 14.

[4] M. Zhu, D. Zhang, J. Zhang, and B. Y. Lim. (2007). Context-Aware Informative Display. International Conference on Multimedia and Expo. Beijing.

[5] A. H. Morales-Aranda and O. Mayora-Ibarra. (2007). A Context Sensitive Public Display for Adaptive Multi-User Information Visualization. Third International Conference on Autonomic and Autonomous Systems. Athens, Greece.

[6] S. Izadi, H. Brignull, T. Rodden, Y. Rogers, and M. Underwood. (2003). Dynamo: A public interactive surface supporting the cooperative sharing and exchange of media. Symposium on User Interface Software and Technology. Vancouver, Canada.

[7] D. M. Russell. (2003). Large interactive public displays: Use patterns, support patterns, community patterns. in Public and Situated Displays: Social and Interactional Aspects of Shared Display Technologies. Kluwer Academic Publishers.

[8] T. Payne, E. David, N. R. Jennings, and M. Sharifi. (2006) Auction Mechanisms for Efficient Advertisement Selection on Public Displays. European Conference on Artificial Intelligence. Riva del Garda, Italy.

[9] J. Müller, A. Schlottmann, and A. Krüger. (2007). Self-optimizing Digital Signage Advertising. Adjunct Proceedings of Ubicomp. Innsbruck.

[10] G. Adomavicius and A. Tuzhilin. (2005). Toward the Next Generation of Recommender Systems: A Survey of the State-of-the-Art and Possible Extensions. in IEEE Transactions on Knowledge and Data Engineering. vol. 17.

[11] F. R. Ribeiro and R. José. (2009) Timeliness for dynamic source selection in situated public displays. 5th Int. Conference on Web Information Systems and Technologies. Lisbon. 